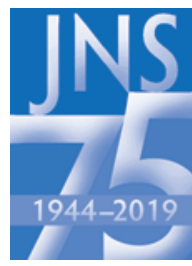

\title{
Clinical trials for pediatric traumatic brain injury: definition of insanity?
}

\author{
JNSPG 75th Anniversary Invited Review Article
}

\author{
Brian Appavu, MD, ${ }^{1,2}$ Stephen T. Foldes, PhD, ${ }^{1}$ and P. David Adelson, MD ${ }^{1,2}$ \\ 'Barrow Neurological Institute at Phoenix Children's Hospital; and 2University of Arizona College of Medicine_Phoenix, \\ Department of Child Health, Phoenix, Arizona
}

\begin{abstract}
Traumatic brain injury (TBI) is a leading cause of morbidity and mortality in children both in the United States and throughout the world. Despite valiant efforts and multiple clinical trials completed over the last few decades, there are no high-level recommendations for pediatric TBI available in current guidelines. In this review, the authors explore key findings from the major pediatric clinical trials in children with TBI that have shaped present-day recommendations and the insights gained from them. The authors also offer a perspective on potential efforts to improve the efficacy of future clinical trials in children following TBI.
\end{abstract}

https://thejns.org/doi/abs/10.3171/2019.2.PEDS18384

KEYWORDS clinical trials; multimodality monitoring; autoregulation; traumatic brain injury; trauma

$\mathrm{T}$ RAUMATIC brain injury (TBI) remains the leading cause of morbidity and mortality in children, affecting an estimated 280 per 100,000 children annually in the United States. ${ }^{26}$ Children hospitalized with severe TBI have a reported mortality rate upwards of $24.2 \% .^{50}$ Those children who survive their initial injuries are susceptible to myriad lifelong complications including posttraumatic epilepsy, cognitive deficits, sleep disorders, psychiatric disorders, and chronic headaches. Overall total life costs of injuries for children with TBI who are younger than 14 years of age have been reported at $\$ 60.4$ billion. ${ }^{19}$ Nearly 500,000 TBI events occur each year and account for more than $70 \%$ of the fatal injuries in children in the United States, ${ }^{21}$ with a similar if not greater clinical burden in other countries. ${ }^{52}$

Despite the significance of this disease, high-level recommendations for pediatric TBI are not available in the current pediatric guidelines ${ }^{31}$ due to a lack of the highquality, successful clinical studies and randomized clinical trials (RCTs) needed for level I recommendations. Clinical studies in both adults and children following severe TBI have largely failed, although similar methodologies for patient categorization, clinical management, and outcomes assessments continue to be used. Is it time for reassessment of our approach to clinical studies?

There is no controversy in the declaration that brain injury is complex. Besides the primary brain injury, numerous pathophysiological processes occur after TBI, including cerebral swelling, seizures, metabolic crises, cortical spreading depolarizations, cerebral vasospasms, and neuroinflammation. ${ }^{35}$ All have the potential to cause subsequent brain damage during the days and weeks subsequent to initial injury. The cumulative burden of these pathophysiological perturbations probably contributes to the cumulative damage incurred and ultimate outcome. With reliance on intracranial pressure (ICP) monitoring alone, does the clinician have enough real-time information of ongoing cerebral injury to make rational decisions? Should clinical management be reassessed with more targeted approaches?

In this review we discuss findings from some of the clinical trials in the pediatric population following TBI that have shaped present-day recommendations and the insights gained from them, and we offer a perspective on future efforts to improve clinical trials in children following TBI.

ABBREVIATIONS ABP = arterial blood pressure; $\mathrm{CPP}=$ cerebral perfusion pressure; $\mathrm{DC}=$ decompressive craniectomy; $\mathrm{GCS}=\mathrm{Glasgow}$ Coma Scale; $\mathrm{GOS}=\mathrm{Glasgow}$ Outcome Scale; GOSE = Glasgow Outcome Scale-Extended; GOSE-Peds = GOSE-Pediatric; ICH = intracranial hypertension; ICP = intracranial pressure; MMM = multimodality monitoring; $\mathrm{PbtO}_{2}=$ partial pressure of brain oxygenation; $\mathrm{PRx}=$ pressure-reactivity index; $\mathrm{RCT}=$ randomized clinical trial; $\mathrm{TBI}=$ traumatic brain injury.

SUBMITTED January 31, 2019. ACCEPTED February 4, 2019.

INCLUDE WHEN CITING DOI: 10.3171/2019.2.PEDS18384. 


\section{Clinical Trials in Severe Pediatric TBI}

Over the past several decades, efforts to define efficacious clinical decisions relative to outcomes after pediatric TBI have been substantial, yet strong evidence remains sparse. In a review of pediatric clinical trials for severe TBI, Natale et al. ${ }^{41}$ noted that through 2005 , only approximately 20 clinical studies existed for children with TBI, with only one being a multicenter $\mathrm{RCT} .{ }^{4}$ Since that publication there have been other new studies, including single-center and multicenter RCTs of medical and surgical interventions to address secondary cerebral edema and intracranial hypertension (ICH). These have served as the evidence base for the publication of the second edition of the guidelines, ${ }^{31}$ although there were many lower-level studies. For the upcoming new edition, ${ }^{32,33}$ a significant number of new studies were added to shape recommendations. Despite 15 years between the first pediatric guidelines and the most recent edition, there remain few level I and some level II recommendations, with the vast majority being level III recommendations due to class III or lower evidence. Although these studies continue to build the foundation for present management recommendations for the treatment of severe TBI, the lack of strong evidence results in inconsistency and variability in these clinical studies. In the first edition ${ }^{3}$ a clinical pathway algorithm was developed among the authors as a consensus of "expert opinion" to provide a basis for consideration in patient management. Yet this clinical management algorithm could only be considered a level III recommendation.

Whereas initiation of standard clinical management includes ensuring oxygenation, cerebral perfusion, and appropriate body temperature, reduction of ICH has been the mainstay of severe TBI management. It has been expected that reducing ICH and the secondary injury response would result in improved outcomes. Unfortunately, this has not been uniformly true. Many studies have focused on treatments targeting cerebral swelling with the goal of effective management of this secondary brain injury phase, yet have fallen short on improving outcomes.

A review of several of these clinical trials may help elucidate the unique aspects of clinical management (Table 1), as well as limitations that have hindered studies from providing higher evidence recommendations. Although these studies may not have achieved their goals, many lessons can be learned. These studies have highlighted numerous problems that potentially inhibit clinical studies in pediatric neurotrauma and doom them to failure from the outset. The review of more recent studies for medical and surgical interventions can be useful to highlight some trial design flaws and perhaps guide improvements in how future trials are conducted.

\section{Hyperosmolar Therapy}

Use of hyperosmolar therapy to reduce ICP has been prevalent over the past 40 years, and both hypertonic saline and mannitol have been used in contemporary ICH management. Mannitol, however, has not been subjected to contemporary controlled clinical trials versus placebo or other therapies in children. Hypertonic saline has also not been rigorously tested except in single-center studies.
Although use of hyperosmolar therapy has been shown to reduce $\mathrm{ICH}$, there has been variability in clinical protocols using each of the therapies (i.e., dosages, timing, etc.) in comparisons between different therapies and in outcome measures used. ${ }^{24,30,47,48}$ Despite a potential effect of mitigating ICH, the results of clinical studies for hyperosmolar therapy have not shown significant improvement in outcomes.

These studies on hyperosmolar therapy have helped contribute to current guidelines at a level II recommendation supporting the bolus use of hypertonic saline (3\%) and at a level III recommendation for bolus of high-dose hypertonic saline $(23.4 \%)$ for the acute treatment of severe ICH. There was also a level III recommendation for the continuous infusion of hypertonic saline for managing ongoing ICH. Note that recommendations were only for ICP control because there was insufficient evidence to give recommendations for improving outcomes.

\section{Decompressive Surgery}

Decompressive craniectomy (DC) has been widely considered to be a second-tier treatment of refractory ICH. Multiple early retrospective studies suggested that timing of DC in relation to brain injury may be relevant and may lead to improved functional outcomes in children..$^{14,43,45,53}$ This notion, however, has not been confirmed in prospective clinical trials. These studies vary in a defined study population, selection criteria for study inclusion, surgical techniques used, and technique for storage and reconstruction of the cranial defect. Notable in these DC studies is the fact that they also are limited by a lack of standardization of follow-up times, variable outcome measures, and small sample sizes. Last, these studies do not directly compare ICP between DC and medical management but rather evaluate ICP and ICH as a secondary outcome measure.

The current TBI guidelines ${ }^{31}$ state that $\mathrm{DC}$ may be considered for pediatric patients with TBI who show early signs of neurological deterioration, herniation, or ICH refractory to medical management. This is a level III recommendation because there is insufficient evidence to make recommendations to improve outcome.

\section{Therapeutic Hypothermia}

Despite vast preclinical evidence that therapeutic hypothermia can prevent or reduce secondary brain injury, ${ }^{1-3,15,36}$ this has not translated to either adult or pediatric clinical studies. Therapeutic hypothermia had been shown to be successful in early phase 2 and 3 single-institution or limited multicenter clinical studies of adults after TBI ${ }^{16}$ and in clinical trials of newborn babies after hypoxic-ischemic encephalopathy, ${ }^{25,46}$ but did not eventually demonstrate efficacy following severe TBI in adults. ${ }^{18}$ Although one adult multicenter phase 3 clinical trial of moderate hypothermia after TBI did not demonstrate overall efficacy, secondary analysis suggested that younger patients tended to have improved outcomes. ${ }^{17}$ Although the initial phase 2 multicenter RCT in pediatric TBI demonstrated that treatment with moderate hypothermia was safe and reduced mortality ${ }^{4}$ this was not confirmed with the later phase 3 multicenter multinational RCT. This study was terminat- 
TABLE 1. Clinical trials for pediatric severe TBI over the past 30 years

\begin{tabular}{lllcll}
\hline \multicolumn{1}{c}{ Authors \& Year } & \multicolumn{1}{c}{ Intervention } & $\begin{array}{c}\text { Study } \\
\text { Quality }\end{array}$ & $\begin{array}{c}\text { No. of } \\
\text { Subjects }\end{array}$ & $\begin{array}{c}\text { Outcome } \\
\text { Measured }\end{array}$ & \multicolumn{1}{c}{ Results } \\
\hline Fisher et al., 1992 & Hypertonic saline (3\%) & Class II & 18 & GOS at 6 mos & Decreased ICP \\
\hline Simma et al., 1998 & Hypertonic saline (1.7\%) & Class II & 35 & Mortality & No difference \\
\hline Taylor et al., 2001 & Decompressive surgery & Class III & 27 & GOS at 6 mos & No difference \\
\hline Adelson et al., 2005 & Hypothermia & Class III & 75 & Complications & No difference \\
\hline Hutchison et al., 2008 & Hypothermia & Class II & 225 & Mortality & Trend to increased mortality \\
\hline Adelson et al., 2013 & Hypothermia & Class II & 77 & Mortality & No difference \\
\hline Beca et al., 2015 & Hypothermia & Class II & 50 & PCPC & No difference \\
\hline
\end{tabular}

$\mathrm{PCPC}=$ Pediatric Cerebral Performance Category

ed early for futility after interim analysis in 77 patients demonstrated no between-group difference in mortality 3 months after injury. ${ }^{5}$ A separate multicenter international trial of 225 children investigated functional outcomes and mortality in children with severe TBI randomized to hypothermia $\left(32.5^{\circ} \mathrm{C}\right.$ for 24 hours) or normothermia groups, ${ }^{28}$ and showed a trend toward higher mortality in the hypothermia group compared to the normothermia group. Last, another international multicenter phase 2 RCT was conducted to assess the feasibility of performing a phase 3 trial of therapeutic hypothermia started early and continued for at least 72 hours in children with severe TBI. There were no differences in outcomes observed. ${ }^{9}$

In all these studies, different variables probably contributed to the lack of positive findings. For example, in 1 study, ${ }^{28}$ differences in clinical management were observed between groups, with the hypothermia group having a significantly increased incidence of systemic hypotension and need for more vasopressors during the rewarming period as compared to normothermic patients. Additionally, these studies suffered from variability in criteria for inclusion between studies and clinical management across studies. Even within a study, there was variability in clinical management across centers, application of therapies, and outcome measures used. ${ }^{5}$ Because of these findings, the severe TBI guidelines state that hypothermia should be avoided in the treatment of pediatric severe TBI to improve outcome, but may be used to reduce ICP as a later-tier therapy at a level III recommendation.

\section{Comparative Effectiveness Research}

Given the challenges observed from traditional interventional RCT to improve outcomes after severe TBI, alternative research strategies emerged. The Approaches and Decisions for Acute Pediatric TBI (ADAPT) trial was designed as an observational cohort study to compare the effectiveness of standard therapies in clinical use and how they impact outcomes. ${ }^{12}$ Having completed enrollment in follow-up, the study will hopefully report as part of its analysis higher levels of recommendation for ICH management, hypoxia management (as measured by partial pressure of brain oxygenation $\left.\left[\mathrm{PbtO}_{2}\right]\right)$, nutritional support, and glucose management, among others. Fifty-one clinical centers from 8 countries participated in patient enrollment with the enrollment of 1000 consecutive patients over 2.5 years. This has already been a productive study through its initial publications. As in other clinical trials, a survey of participating sites found that there were significant variations of medical goals that exist between sites, variable neurophysiological targets, and different strategies to treat those targets for all trial hypotheses tested. ${ }^{11}$ Initial analyses on the study subjects enrolled has already validated that Glasgow Coma Scale (GCS) scores incrementally predicted mortality, ${ }^{39}$ but that variations exist between children who suffered accidental and abusive head trauma. ${ }^{38}$ Data from this study are expected to begin to appear in published literature in the next few years; enrolled patients have all concluded their last outcome time points. Through this research, we expect there will be greater understanding of present-day clinical management and that the findings will potentially identify optimal clinical approaches for specific patients and directions for future studies.

\section{Lessons That Might Shape Future Trials}

In this day of precision medicine, we have made great strides in our understanding of numerous diseases. With the lack of significant progress regarding pediatric TBI, it is necessary to consider new approaches that may include a more precision-based approach to clinical studies. As mentioned, significant inter- and intrainstitutional variability in inclusion criteria, clinical management, and between-study differences as well as a lack of sensitivity and specificity in the outcome measures used have resulted in the lack of high-level evidence to guide therapy. Is this a "Catch-22," in which we cannot obtain better evidence until we develop better studies and better studies need to be designed without good preliminary evidence? New approaches to diagnosis, clinical management, outcome measures, and implementation of new technologies may help to reenergize new clinical trials to improve care.

\section{Categorization of TBI}

Clinical management of brain tumors is no longer solely based on histological criteria or malignancy status. In contrast, the categorization of TBI severity for the last 40 years has been based on the GCS as the gold standard indicator of injury level. Although there have been modifications to the GCS for the pediatric population, because the original scoring system did not account for developmental issues, ${ }^{12,37}$ the GCS may still not be applicable throughout the age spectrum and with variability in injury mechanisms 
incurred after TBI. The GCS has also been questioned in studies conducted in adult patients, given that scores do not adequately reflect the magnitude and quality of injury pathology and are not enough to group similar patients. A GCS score of 4 could include patients with an epidural hematoma, subdural hematoma, intraparenchymal contusions, subarachnoid hemorrhage, diffuse axonal injury, hypoxic-ischemic encephalopathy, or combinations of all these processes. Different injuries may represent unique pathologies that could vary in their response to treatment modalities. Through experience, we know that a patient with a presenting GCS score of 4 who has a pure epidural hematoma, and even a unilaterally dilated pupil, will do well with aggressive surgical management and will be unlikely to have later ICH and need for significant medical management. In contrast, a patient with a GCS score of 4 but with diffuse cerebral contusions, bilateral subdural hematomas, and cerebral edema with midline shift will probably require aggressive medical care and possibly surgical intervention.

Because brain tumors are now subcategorized using several tumor-specific markers prior to study inclusion, the question arises whether it is necessary to begin to do the same for TBI-to subcategorize TBIs so that similar pathologies can be tested against present and future therapies. For the diagnosis of brain tumors, neuroimaging, histological appearance, genotype, and tumor-specific biomarkers have all been used as inclusion criteria prior to interventional research and clinical management. For TBI, to apply principles of precision medicine, this may require expanding our categorization of patients beyond the GCS. TBI-specific biomarkers could be developed to delineate the overall "biosignature" of an individual child to appropriately categorize similar TBI pathologies. Based on present knowledge in addition to the GCS, these biomarkers could include other parameters such as initial imaging (i.e., Marshall classification); neurological examination (i.e., pupillary dilatation); serum biomarkers indicative of neural injury (i.e., glial fibrillary acidic protein, S100B, neuronspecific enolase); and genotype (i.e., APO E4 allele, etc.). With subcategorization, similar pathologies could be tested with targeted therapies in smaller clinical trials with homogeneous populations.

\section{Outcomes Assessments}

It is unexpected that complex cerebral functions could be easily categorized (as is typical in TBI clinical trials) by using a simple 5-point Glasgow Outcome Scale (GOS) score or the 8-point Glasgow Outcome Scale-Extended (GOSE) score. The GOSE-Pediatric (GOSE-Peds) was modified for children ${ }^{10}$ but has the same inherent issues as adult functional scales. Other outcomes assessments have included the Pediatric Cerebral Performance Category scale $^{23}$ and the Pediatric Overall Performance Category scale, ${ }^{44}$ which use similar categories to the GOS (mild, moderate, and severe disability) and the Rancho Los Amigos scale ${ }^{20}$ that are global in application. With variability in injury and postinjury findings, there can be significant differences in the functional capabilities between patients with similar outcome scores. A patient with a predominant injury to the nondominant somatosensory cortex may have functional motor difficulties requiring assistance for activities of daily living but will otherwise be cognitively intact and function at a high level. Nevertheless, such a patient may still carry a GOSE-Peds score indicative of severe disability. In contrast, the patient who can get dressed and feed him- or herself but suffered damage to the fornices or dominant temporal lobe would have difficulty in memory function and/or language and carry a GOSE-Peds severe disability score as well. These 2 patients clearly represent different injuries and outcomes yet have similar outcome scores.

Subtle differences exist that are not easily measured with our present outcome scales. To delineate these subtler outcomes, more comprehensive neuropsychological testing is available but is not often used as part of outcome measures in clinical trials because of the complex report scoring and interpretation. The challenge for investigators involved in clinical trials is to make comparisons of specific neuropsychological subscores and develop relevant outcome measures without requiring large patient numbers to show treatment effect. It is likely that other new outcome scales with specific targeted neural functional subscores will be needed to assess patients based on their pathology. Additionally, it would be beneficial to have an overall score to better define the relationship between initial diagnosis and categorization through clinical management rather than outcomes assessment alone.

\section{Advanced Monitoring in Severe TBI Challenges}

The current guidelines for pediatric severe TBI demonstrate our inadequate knowledge regarding the efficacy of existing treatment paradigms. ${ }^{31}$ This paucity of evidence is probably related to the complex pathophysiological processes that underlie TBI, including high variability in primary injury mechanisms and heterogeneous secondary insults..$^{34}$ Idiosyncrasies of the molecular, cellular, and physiological responses to the initial insult are potential confounders, and are probably more impactful within the developing brain. Prior clinical trials that targeted hyperosmolar, brain compliance, and hypothermic therapies were predicated on a foundation of known pathophysiological mechanisms, retrospective analyses, and bench research that suggested potential benefit. The inability to translate this work into successful human interventional trials may be related to our historical inability to truly monitor the pathophysiological complexities of disease evolution in the clinical environment. A clear need exists to develop better bedside methodologies to characterize a child's physiological state and stratify their risk of injury and outcomes.

The vast majority of neurocritical care for the treatment of severe TBI has focused on the clinical management of ICP and the prevention or mitigation of ICH. If the focus is on decreasing ICP beyond a threshold, the brain has already swollen past a threshold for management (i.e., 20 $\mathrm{mm} \mathrm{Hg}$ ). Traditional management of these patients is a dichotomous treatment decision tree of "Yes," we treat above threshold, or "No," we do not treat below threshold. This simplified approach to managing the complex processes of acute brain injury ignores other latent pathophysiological 


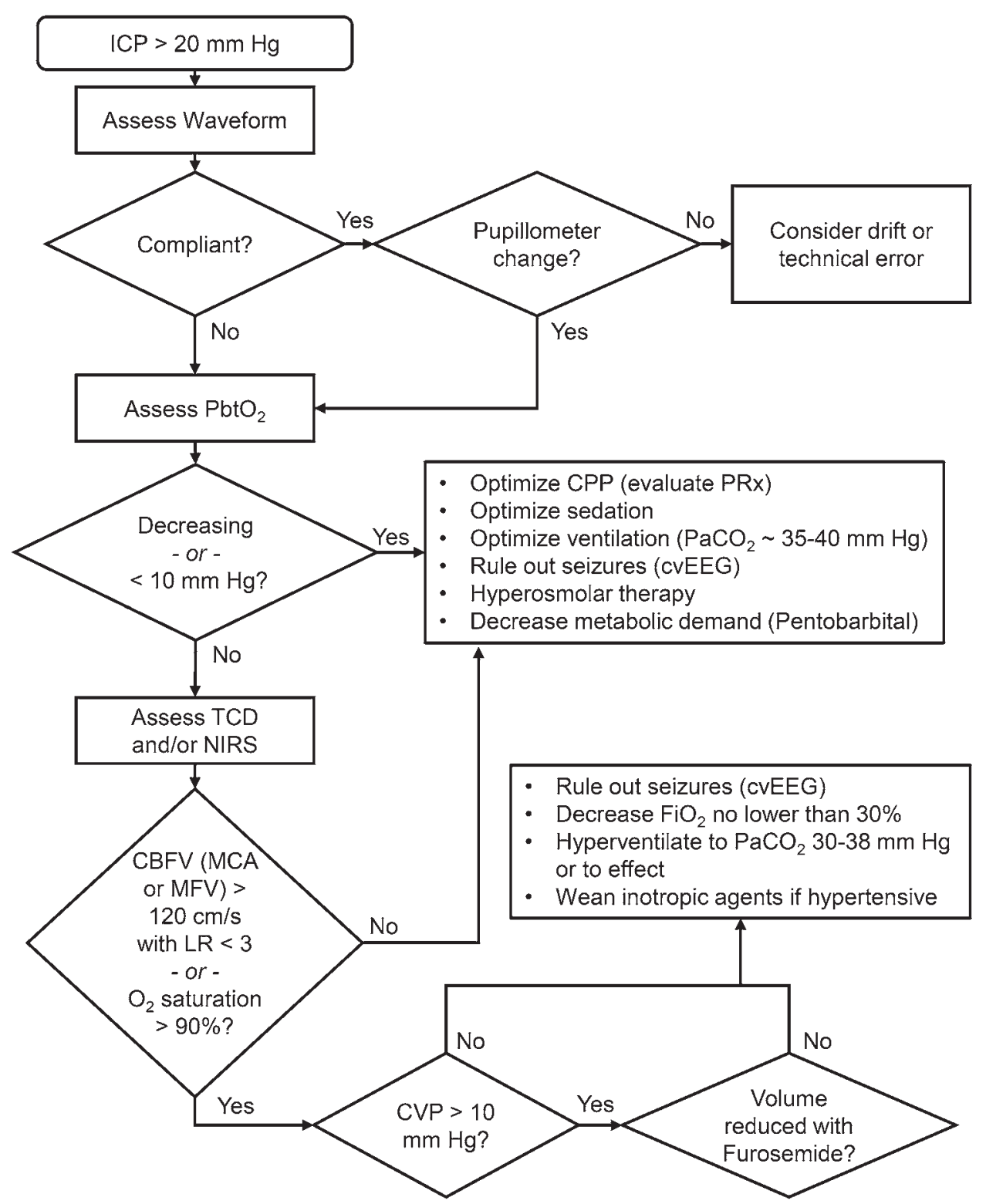

FIG. 1. Example of a clinical decision-making algorithm designed using MMM data. CBFV = cerebral blood flow volume; cvEEG = continuous video EEG; CVP = central venous pressure; $L R=$ Lindengaard ratio; $M C A$ = middle cerebral artery; $M F V=$ mean flow velocity; NIRS = near-infrared spectroscopy; $T C D=$ transcranial Doppler.

processes that occur in the postinjury phase. Adding other measures of cerebral physiology (i.e., brain oxygenation) provides a deeper understanding of real-time, complex physiological processes and provides new opportunities for expanding the decision tree for clinical management (Fig. 1). In the clinical scenario that ICP is greater than $20 \mathrm{~mm}$ $\mathrm{Hg}$, there can be multiple etiologies contributing to that elevated pressure. If a $\mathrm{PbtO}_{2}$ monitor is used in conjunction with the measurement of ICP and $\mathrm{PbtO}_{2}$ is elevated, this would indicate excessive perfusion relative to metabolism. In this scenario, elevated ICP is probably secondary to hyperemia with subsequent vasogenic edema. In contrast, when $\mathrm{PbtO}_{2}$ is low, indicating ischemia, the pathophysiology of that elevated ICP would more likely be due to cytotoxic edema. Clinical management for these 2 situations might differ, with targeted therapies that lower cerebral blood flow used in the first scenario but other therapies to potentially increase cerebral blood flow used in the second scenario. Our present clinical management with singlemodality monitoring would not make the distinction between these 2 pathophysiological situations.

\section{Multimodality Monitoring}

To address the heterogeneity of the acutely injured brain following TBI, there are many dynamic and complex pathophysiological events that require measurement through monitoring strategies that provide new potential interventions to preserve injured but recoverable brain tissue. With the development of advanced neurological monitoring technologies, there is potential to provide pivotal and real-time information to improve decision-making in neurocritical care. Real-time bedside information can be 

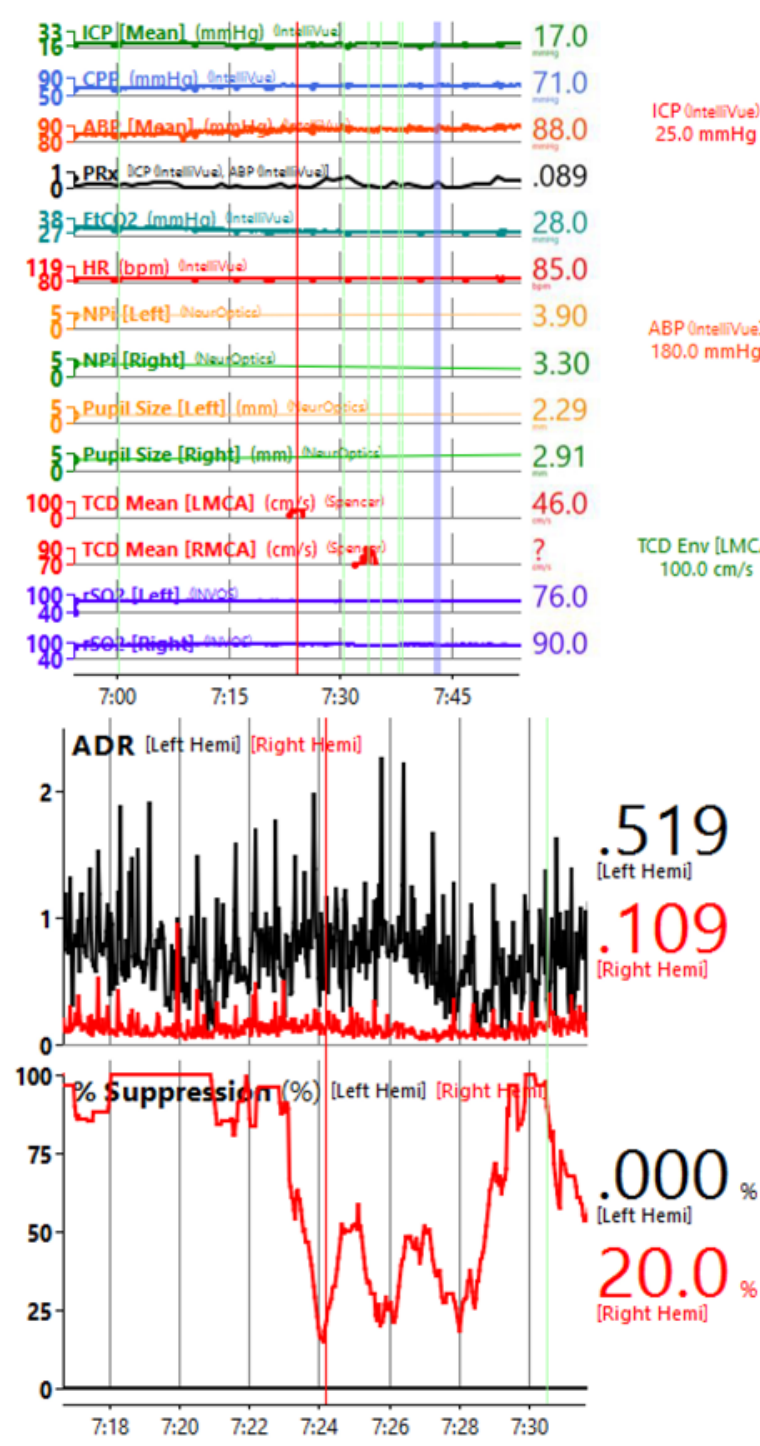

FIG. 2. Example of MMM visualization platform with physiological trend and waveform analysis (Moberg CNSReader; Moberg ICU Solution).

used to assess the "functional health" of the brain through measurement of physiological processes that mediate secondary brain injury, assess efficacy of therapeutic interventions, and personalize approaches to care. There are a few adult studies in which $\mathrm{PbtO}_{2}$ monitoring was used with early positive results. ${ }^{42}$ In the current pediatric guidelines, there were only 2 class III prospective noninterventional cohort studies that used $\mathrm{PbtO}_{2}$ in conjunction with ICP monitoring in children following severe TBI to assess outcomes. ${ }^{22,40}$ This work only showed that it was a safe modality and that low $\mathrm{PbtO}_{2}$ correlated with poor outcome. Evidence from monitoring in children with other invasive and noninvasive monitoring technologies besides those for ICP and $\mathrm{PbtO}_{2}$ is limited yet emerging.

For complex pathophysiological processes that occur after brain injury, it is likely that measurement and simple reporting of neurophysiological data will be insufficient. Ultimately, the hypothesis-driven understanding of a patient's unique physiological biosignature will help guide clinical management. To optimize the ability to visualize and correlate different physiological processes, technologies have been advanced to collect data from numerous monitoring devices in a continuous, time-synchronized manner and to set the foundation for more integrated approaches. This synchronized data aggregation or multimodality monitoring (MMM) of physiological parameters can occur in high temporal resolution in dashboards that allow one to explore trends in continuous quantitative values for physiological variables such as ICP or $\mathrm{PbtO}_{2}$, as well as analysis of time-synchronized waveforms of ICP, transcranial Doppler ultrasound flow velocities, or EEG? Concurrent raw waveforms can be observed as well, allowing for identification of changing physiology as well as potential artifact (Fig. 2). From such trends, clinicians may be able to envision various pathophysiological states at any given time in each patient and derive the etiology of specific physiological disturbances. This may provide unique opportunities for novel therapeutic approaches. 

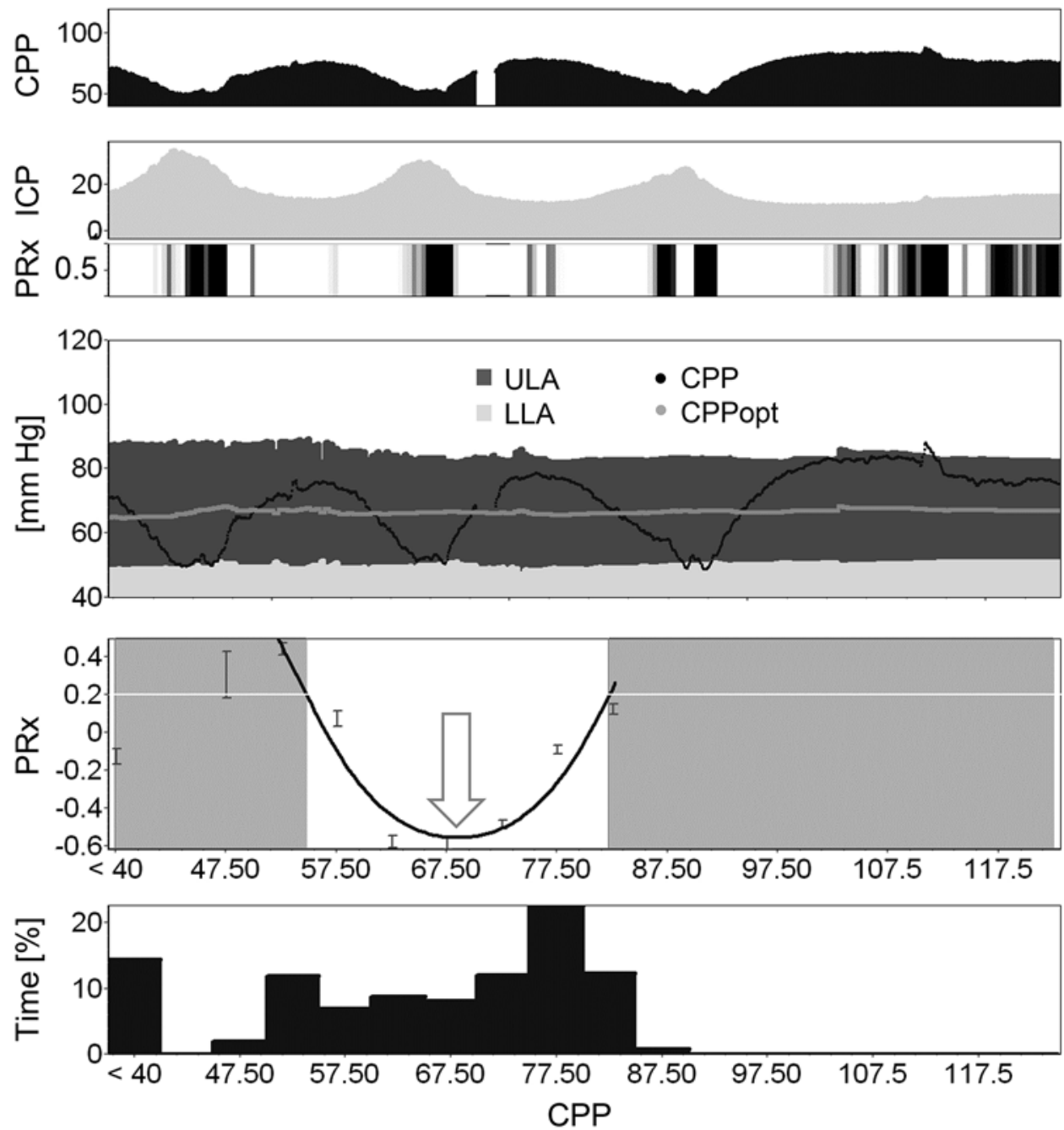

FIG. 3. Evaluation of cerebral autoregulation from MMM data. Continuous physiological data can be used to visualize and understand model-based indices of cerebral autoregulation. In this example, the PRx is determined by calculating a Pearson correlation coefficient computation of ICP and mean ABP (ICM+, Cambridge Enterprises). By plotting the PRx over a range of CPPs, one can determine the optimal CPP (CPPopt) by assessing the CPP at which the PRx is most negative (arrow). In this case of a child with severe TBI, the CPPopt is calculated at $68.6 \mathrm{~mm} \mathrm{Hg}$. LLA = lower limit of autoregulation; ULA = upper limit of autoregulation.

\section{Analytical Approaches to MMM}

With aggregation and synchronization of various physiological data by using MMM, advanced computational model-based indices can be analyzed describing cerebral autoregulation, hemodynamics, brain compliance, and entropy measures. ${ }^{54}$ The pressure-reactivity index $(\mathrm{PRx}),{ }^{55}$ mean velocity index,,$^{13}$ oxygen reactivity index,${ }^{29}$ and cerebral oxygenation index ${ }^{51}$ represent several new indices being used in clinical practice to assess cerebral autoregulation through the changes in ICP, flow velocity, arterial blood pressure (ABP), $\mathrm{PbtO}_{2}$, and near-infrared spectroscopy oxygen saturation. These indices use dynamic linear regression analyses to formulate correlation coefficients within a moving window. Optimal physiological states can then be determined by observing these indices over a range of conditions and changing parameters that can guide clinical management. With thousands of time points correlated between cerebral perfusion pressure (CPP), ICP, and ABP, an "optimal CPP" can be formulated by evaluating the
PRx over a CPP range and guiding the lower threshold for CPP to maintain an optimal autoregulatory state (Fig. 3). ${ }^{8}$

These early relational computational models will probably play a much larger role in clinical management with further advancements in bioinformatics and machine learning. Advanced statistics, time series analyses, and data mining will soon offer useful tools for the investigation and clinical use of MMM data. Machine learning algorithms have been used to analyze electronic medical records and predict outcomes in TBI. ${ }^{27}$ Decision-tree analysis allows for a set of variables to be grouped together, then subdivided into differential characteristics and analyzed on specific outcomes. This technique has been used to identify subgroups of adults with head injuries who go on to have a poor prognosis. ${ }^{6}$ Cluster analysis can also be used to discern inherent patterns in multimodal physiological data. Using this technique, perturbed local chemistry detected on cerebral microdialysis was found to be associated with varied metabolic states. With weak correlations 
to $\mathrm{CPP}$ and ICP in adults with TBI, distinct physiological profiles could be identified among patients with TBI who died, contracted an infection, and suffered multiple organ failure. ${ }^{49}$ Clearly, further work is necessary, but these early studies provide a window into the potential for improved understanding of the complex neurophysiology following severe TBI in children and opportunities for more aggressive and novel therapeutic approaches for clinical management.

\section{Conclusions}

Despite tremendous effort, there has been limited progress in validating the efficacy of interventions to improve outcomes in children with severe TBI. Previous clinical trials were often hindered by variability and heterogeneity of age and injury in the pediatric population, variability in the medical and surgical interventions across and within centers, and variability in type and reliability of outcome measures. With growth of the technology to provide higher levels of neurointensive care with a better understanding of real-time brain physiology, there is potential for improved identification of specific physiological states after pediatric TBI. Further advances in MMM may offer new insights into the complex pathophysiology of pediatric TBI and facilitate identification of patient subgroups through biosignatures that may lead to new treatment targets. Improved ways to stratify patients and monitor and manage their care with targeted measures could strengthen future clinical trials.

\section{Acknowledgments}

This work was supported by a research grant from Integra Codman Franchise for program development.

\section{References}

1. Adelson PD: Animal models of traumatic brain injury in the immature: a review. Exp Toxicol Pathol 51:130-136, 1999

2. Adelson PD: Hypothermia following pediatric traumatic brain injury. J Neurotrauma 26:429-436, 2009

3. Adelson PD, Bratton SL, Carney NA, Chestnut RM, du Coudray HE, Goldstein B, et al: Guidelines for the acute medical management of severe traumatic brain injury in infants, children and adolescents. Pediatr Crit Care Med 4 (3 Suppl):S38-S39, 2003

4. Adelson PD, Ragheb J, Kanev P, Brockmeyer D, Beers SR, Brown SD, et al: Phase II clinical trial of moderate hypothermia after severe traumatic brain injury in children. Neurosurgery 56:740-754, 2005

5. Adelson PD, Wisniewski SR, Beca J, Brown SD, Bell M, Muizelaar JP, et al: Comparison of hypothermia and normothermia after severe traumatic brain injury in children (Cool Kids): a phase 3, randomised controlled trial. Lancet Neurol 12:546-553, 2013

6. Andrews PJ, Sleeman DH, Statham PF, McQuatt A, Corruble $\mathrm{V}$, Jones PA, et al: Predicting recovery in patients suffering from traumatic brain injury by using admission variables and physiological data: a comparison between decision tree analysis and logistic regression. J Neurosurg 97:326-336, 2002

7. Appavu B, Riviello JJ: Electroencephalographic patterns in neurocritical care: pathologic contributors or epiphenomena? Neurocrit Care 29:9-19, 2018

8. Aries MJ, Czosnyka M, Budohoski KP, Steiner LA, Lavinio
A, Kolias AG, et al: Continuous determination of optimal cerebral perfusion pressure in traumatic brain injury. Crit Care Med 40:2456-2463, 2012

9. Beca J, McSharry B, Erickson S, Yung M, Schibler A, Slater A, et al: Hypothermia for traumatic brain injury in children-a phase II randomized controlled trial. Crit Care Med 43:1458-1466, 2015

10. Beers SR, Wisniewski SR, Garcia-Filion P, Tian Y, Hahner $T$, Berger RP, et al: Validity of a pediatric version of the Glasgow Outcome Scale-Extended. J Neurotrauma 29:1126-1139, 2012

11. Bell MJ, Adelson PD, Hutchison JS, Kochanek PM, Tasker RC, Vavilala MS, et al: Differences in medical therapy goals for children with severe traumatic brain injury-an international study. Pediatr Crit Care Med 14:811-818, 2013

12. Bell MJ, Adelson PD, Wisniewski SR: Challenges and opportunities for pediatric severe TBI-review of the evidence and exploring a way forward. Childs Nerv Syst 33:1663-1667, 2017

13. Budohoski KP, Reinhard M, Aries MJ, Czosnyka Z, Smielewski P, Pickard JD, et al: Monitoring cerebral autoregulation after head injury. Which component of transcranial Doppler flow velocity is optimal? Neurocrit Care 17:211-218, 2012

14. Cho DY, Wang YC, Chi CS: Decompressive craniotomy for acute shaken/impact baby syndrome. Pediatr Neurosurg 23:192-198, 1995

15. Clark RS, Kochanek PM, Marion DW, Schiding JK, White M, Palmer AM, et al: Mild posttraumatic hypothermia reduces mortality after severe controlled cortical impact in rats. J Cereb Blood Flow Metab 16:253-261, 1996

16. Clifton GL, Allen S, Barrodale P, Plenger P, Berry J, Koch S, et al: A phase II study of moderate hypothermia in severe brain injury. J Neurotrauma 10:263-271, 273, 1993

17. Clifton GL, Miller ER, Choi SC, Levin HS, McCauley S, Smith KR Jr, et al: Lack of effect of induction of hypothermia after acute brain injury. N Engl J Med 344:556-563, 2001

18. Clifton GL, Valadka A, Zygun D, Coffey CS, Drever P, Fourwinds S, et al: Very early hypothermia induction in patients with severe brain injury (the National Acute Brain Injury Study: Hypothermia II): a randomised trial. Lancet Neurol 10:131-139, 2011

19. Corso P, Finkelstein E, Miller T, Fiebelkorn I, Zaloshnja E: Incidence and lifetime costs of injuries in the United States. Inj Prev 12:212-218, 2006

20. Duclos C, Dumont M, Arbour C, Paquet J, Blais H, Menon DK, et al: Parallel recovery of consciousness and sleep in acute traumatic brain injury. Neurology 88:268-275, 2017

21. Faul M, Xu L, Wald MM, Coronado VG: Traumatic Brain Injury in the United States: Emergency Department Visits, Hospitalizations and Deaths 2002-2006. Atlanta: Centers for Disease Control and Prevention, National Center for Injury Prevention and Control, 2010

22. Figaji AA, Zwane E, Thompson C, Fieggen AG, Argent AC, Le Roux PD, et al: Brain tissue oxygen tension monitoring in pediatric severe traumatic brain injury. Part 1: Relationship with outcome. Childs Nerv Syst 25:1325-1333, 2009

23. Fiser DH, Tilford JM, Roberson PK: Relationship of illness severity and length of stay to functional outcomes in the pediatric intensive care unit: a multi-institutional study. Crit Care Med 28:1173-1179, 2000

24. Fisher B, Thomas D, Peterson B: Hypertonic saline lowers raised intracranial pressure in children after head trauma. $\mathbf{J}$ Neurosurg Anesthesiol 4:4-10, 1992

25. Gunn AJ: Cerebral hypothermia for prevention of brain injury following perinatal asphyxia. Curr Opin Pediatr 12:111-115, 2000

26. Hawley CA, Ward AB, Long J, Owen DW, Magnay AR: Prevalence of traumatic brain injury amongst children ad- 
mitted to hospital in one health district: a population-based study. Injury 34:256-260, 2003

27. Hemphill JC, Andrews P, De Georgia M: Multimodal monitoring and neurocritical care bioinformatics. Nat Rev Neurol 7:451-460, 2011

28. Hutchison JS, Ward RE, Lacroix J, Hébert PC, Barnes MA, Bohn DJ, et al: Hypothermia therapy after traumatic brain injury in children. N Engl J Med 358:2447-2456, 2008

29. Jaeger M, Schuhmann MU, Soehle M, Meixensberger J: Continuous assessment of cerebrovascular autoregulation after traumatic brain injury using brain tissue oxygen pressure reactivity. Crit Care Med 34:1783-1788, 2006

30. Khanna S, Davis D, Peterson B, Fisher B, Tung H, O'Quigley $\mathrm{J}$, et al: Use of hypertonic saline in the treatment of severe refractory posttraumatic intracranial hypertension in pediatric traumatic brain injury. Crit Care Med 28:1144-1151, 2000

31. Kochanek PM, Carney N, Adelson PD, Ashwal S, Bell MJ, Bratton S, et al: Guidelines for the acute medical management of severe traumatic brain injury in infants, children, and adolescents - second edition. Pediatr Crit Care Med 13 (Suppl 1):S1-S82, 2012

32. Kochanek PM, Tasker RC, Bell MJ, Adelson PD, Carney N, Vavilala MS, et al: Management of pediatric severe traumatic brain injury: 2019 consensus and guidelines-based algorithm for first and second tier therapies. Pediatr Crit Care Med 20:269-279, 2019

33. Kochanek PM, Tasker RC, Carney N, Totten AM, Adelson PD, Selden NR, et al: Guidelines for the Management of Pediatric Severe Traumatic Brain Injury, Third Edition: Update of the Brain Trauma Foundation Guidelines. Pediatr Crit Care Med 20 (Suppl 1):S1-S82, 2019

34. Maas AI, Stocchetti N, Bullock R: Moderate and severe traumatic brain injury in adults. Lancet Neurol 7:728-741, 2008

35. Maas AIR, Menon DK, Adelson PD, Andelic N, Bell MJ, Belli A, et al: Traumatic brain injury: integrated approaches to improve prevention, clinical care, and research. Lancet Neurol 16:987-1048, 2017

36. Mansfield RT, Schiding JK, Hamilton RL, Kochanek PM: Effects of hypothermia on traumatic brain injury in immature rats. J Cereb Blood Flow Metab 16:244-252, 1996

37. Marion DW, Carlier PM: Problems with initial Glasgow Coma Scale assessment caused by prehospital treatment of patients with head injuries: results of a national survey. $\mathbf{J}$ Trauma 36:89-95, 1994

38. Miller Ferguson N, Sarnaik A, Miles D, Shafi N, Peters MJ, Truemper E, et al: Abusive head trauma and mortality-an analysis from an international comparative effectiveness study of children with severe traumatic brain injury. Crit Care Med 45:1398-1407, 2017

39. Murphy S, Thomas NJ, Gertz SJ, Beca J, Luther JF, Bell MJ, et al: Tripartite stratification of the Glasgow Coma Scale in children with severe traumatic brain injury and mortality: an analysis from a multi-center comparative effectiveness study. J Neurotrauma [epub ahead of print], 2017

40. Narotam PK, Burjonrappa SC, Raynor SC, Rao M, Taylon C: Cerebral oxygenation in major pediatric trauma: its relevance to trauma severity and outcome. J Pediatr Surg 41:505-513, 2006

41. Natale JE, Joseph JG, Pretzlaff RK, Silber TJ, Guerguerian AM: Clinical trials in pediatric traumatic brain injury: unique challenges and potential responses. Dev Neurosci 28:276-290, 2006

42. Okonkwo DO, Shutter LA, Moore C, Temkin NR, Puccio AM, Madden CJ, et al: Brain Oxygen Optimization in Severe Traumatic Brain Injury Phase-II: a phase II randomized trial. Crit Care Med 45:1907-1914, 2017

43. Polin RS, Shaffrey ME, Bogaev CA, Tisdale N, Germanson T, Bocchicchio B, et al: Decompressive bifrontal craniectomy in the treatment of severe refractory posttraumatic cerebral edema. Neurosurgery 41:84-94, 1997
44. Pollack MM, Holubkov R, Funai T, Clark A, Moler F, Shanley T, et al: Relationship between the functional status scale and the pediatric overall performance category and pediatric cerebral performance category scales. JAMA Pediatr 168:671-676, 2014

45. Ruf B, Heckmann M, Schroth I, Hügens-Penzel M, Reiss I, Borkhardt A, et al: Early decompressive craniectomy and duraplasty for refractory intracranial hypertension in children: results of a pilot study. Crit Care 7:R133-R138, 2003

46. Shankaran S, Laptook AR, Ehrenkranz RA, Tyson JE, McDonald SA, Donovan EF, et al: Whole-body hypothermia for neonates with hypoxic-ischemic encephalopathy. N Engl J Med 353:1574-1584, 2005

47. Shein SL, Ferguson NM, Kochanek PM, Bayir H, Clark RS, Fink EL, et al: Effectiveness of pharmacological therapies for intracranial hypertension in children with severe traumatic brain injury-results from an automated data collection system time-synched to drug administration. Pediatr Crit Care Med 17:236-245, 2016

48. Simma B, Burger R, Falk M, Sacher P, Fanconi S: A prospective, randomized, and controlled study of fluid management in children with severe head injury: lactated Ringer's solution versus hypertonic saline. Crit Care Med 26:1265-1270, 1998

49. Sinha S, Hudgins E, Schuster J, Balu R: Unraveling the complexities of invasive multimodality neuromonitoring. Neurosurg Focus 43(5):E4, 2017

50. Stanley RM, Bonsu BK, Zhao W, Ehrlich PF, Rogers AJ, Xiang H: US estimates of hospitalized children with severe traumatic brain injury: implications for clinical trials. Pediatrics 129:e24-e30, 2012

51. Suzuki S, Takasaki S, Ozaki T, Kobayashi Y: A tissue oxygenation monitor using NIR spatially resolved spectroscopy. Proc SPIE 3597:582-592, 1999

52. Tasker RC, Fleming TJ, Young AE, Morris KP, Parslow RC: Severe head injury in children: intensive care unit activity and mortality in England and Wales. Br J Neurosurg 25:6877, 2011

53. Taylor A, Butt W, Rosenfeld J, Shann F, Ditchfield M, Lewis $\mathrm{E}$, et al: A randomized trial of very early decompressive craniectomy in children with traumatic brain injury and sustained intracranial hypertension. Childs Nerv Syst 17:154162,2001

54. Varsos GV, Kasprowicz M, Smielewski P, Czosnyka M: Model-based indices describing cerebrovascular dynamics. Neurocrit Care 20:142-157, 2014

55. Zweifel C, Lavinio A, Steiner LA, Radolovich D, Smielewski P, Timofeev I, et al: Continuous monitoring of cerebrovascular pressure reactivity in patients with head injury. Neurosurg Focus 25(4):E2, 2008

\section{Disclosures}

The authors report no conflict of interest concerning the materials or methods used in this study or the findings specified in this paper.

\section{Author Contributions}

Conception and design: all authors. Acquisition of data: all authors. Analysis and interpretation of data: all authors. Drafting the article: all authors. Critically revising the article: all authors. Reviewed submitted version of manuscript: all authors. Approved the final version of the manuscript on behalf of all authors: Adelson.

\section{Correspondence}

P. David Adelson: Barrow Neurological Institute at Phoenix Children's Hospital, Phoenix, AZ. dadelson@phoenixchildrens.com. 